\title{
2018 Acknowledgment of Reviewers
}

A peer-reviewed journal cannot succeed without the dedication and insightful critiques of the reviewers whose efforts often go unrecognized. The editorial leadership of Pediatric Allergy, Immunology, and Pulmonology is very grateful for the support of our expert peer reviewers who have donated their valuable time in 2018 . We thank you for your service.

\begin{tabular}{|c|c|c|}
\hline Roheena Abdullah & Kelly Catapano & Luz Fonacier \\
\hline Stuart Abramson & Prof. Gülfem Çelik & Erick Forno \\
\hline Darryl Adamko & H. Che & G. C. Forte \\
\hline Butch Adolph & Jonathan Cogen & Bengt Francisco \\
\hline Dritan Agalliu & Antonio Condino-Neto & Jonathan Gaffin \\
\hline Tarig Ali-Dinar & John Crane & Andrea Gentili \\
\hline Catarina Amqvist & Paolo Criado & Brian Gilchrist \\
\hline Traci Arney & L. Cuthbertson & Matthew Giuca \\
\hline Miriam Ba & Enza D'Auria & C. Glasser \\
\hline Ian M. Balfour-Lynn & J. Dara & Y. Goto \\
\hline Safa Baris & Michael Davis & Karen Gregory \\
\hline A. Beigelman & Belen de la Hoz & Claudia Guglielmo \\
\hline Y. Belessis & J. Deerojanawong & Ernst Gundula \\
\hline Luca Bertelli & Maria Elisa DiCicco & R. Gupta \\
\hline A. Bidiwala & Endy Dominguez & E. Halvorson \\
\hline Kathryn Blake & M. Donadio & R. D. Hardy \\
\hline Miguel Blanca & Henry Dorkin & Koray Harmanci \\
\hline Faris F. Brkic & P. E. Draber & Kathleen Harrington \\
\hline Ana Broyles & Ana Duarte & Rashed Hasan \\
\hline Ronna Campbell & Nemr Eid & Catherine Hawrylowicz \\
\hline Demet Can & Doaa El Amrousy & Linda Herbert \\
\hline P. Capucilli & R. England & Farhad Heydarian \\
\hline Thomas Caraccio & Hossein Esmaeilzadeh & Michael Hines \\
\hline Christopher Carroll & Harold Farber & Xiumei Hong \\
\hline Jose Castro-Rodriguez & Ronald Ferdman & T. Hoshina \\
\hline
\end{tabular}


Daniel Hsu

S. K. Huang

Katrina Hutchison

Erik Hysinger

C. Inuo

Shahidul Islam

K. Izukara

Thomas Janssens

Christine Joseph

Stephanie Jouneau
A. Karim
M. Kazachov
Bill Kelly
H. Kelly
John Kelso
Ozlem Keskin
Sara Khan
Catherine Kier
Jon Konradsen
Anastassios Koumbourlis
M. Krause
Cadence Kuklinski
Simon Langton Hewer
Men-Jean Lee
Burt Lesnick
Amelia Licari
Kenneth Lieuw
Eric Lloyd
Catherine Lok
V. Lougaris
Isabelle Lowell
Evans Machogu
Eric Macy

A. Maitland van der Zee

Jonathan Mansbach

Lijun Mao

Tuvia Marciano

G. L. Marseglia

Margaret McCormick

P. Mendoza

Karen Meyerson

Susan Millard

Emily Muther

S. Nadel

Josh Needleman

P. Nguyen

Asif Noor

Cade Nylund

F. Obuchi

Celso Eduardo Oliver

Nicholas Osborne

R. Padoan

Giovanni Pajno

E. Papadopolou-Alataki

H. J. Park

Sevgi Pekcan

Hanna Phan

Claude Ponvert

Jonathan Popler

Dejan Radovanovic

Hengameh Raissy

Clement Ren

W. Rigby

C. E. Rodriguez-Martinez

Ray Rodriguez

M. Roth
Bruce K. Rubin

U. Sack

Helton Santiago

Maria Santiago

E. Savage

Bianca Schaub

Petra Schelstaete

M. Sears

Ahmet Sert

N. Shah

Rasik Shah

Rasik Shah

Shaharyar Sheikh

Robert Silverman

Florian Singer

T. Soyer

Shiro Sugiura

Dong In Suh

Stanley Szefler

V. Tangpricha

Xinrong Tao

Bianca Tesi

Hector Thomson

Alberto Tommasini

Ahmet Turkeli

Kazuhiro Uda

Naveen Uli

R. Uotila

Julia Upton

Pinar Uysal

A. Viagea

R. Viera

Susanne Vijverberg 
Volker Wahn

Linding Wang

M. Wang

Min Wang

Miles Weinberger
Daniel Weiner

Michael Welch

Dennis Williams

Pingsheng Wu

Z. Xiang
Aravind Yadav

Q. Ye

Z. Zafari

Anna Zolin 